\title{
Portable Vector Flow Imaging Compared with Spectral Doppler Ultrasonography
}

di lanni, Tommaso; Hansen, Kristoffer Lindskov; Villagómez Hoyos, Carlos Armando; Moshavegh, Ramin; Bachmann Nielsen, Michael; Jensen, Jørgen Arendt

Published in:

I E E E Transactions on Ultrasonics, Ferroelectrics and Frequency Control

Link to article, DOI:

10.1109/TUFFC.2018.2872508

Publication date:

2018

Document Version

Peer reviewed version

Link back to DTU Orbit

Citation $(A P A)$ :

di lanni, T., Hansen, K. L., Villagómez Hoyos, C. A., Moshavegh, R., Bachmann Nielsen, M., \& Jensen, J. A. (2018). Portable Vector Flow Imaging Compared with Spectral Doppler Ultrasonography. I E E E Transactions on Ultrasonics, Ferroelectrics and Frequency Control, 66(3), 453 - 462.

https://doi.org/10.1109/TUFFC.2018.2872508

\section{General rights}

Copyright and moral rights for the publications made accessible in the public portal are retained by the authors and/or other copyright owners and it is a condition of accessing publications that users recognise and abide by the legal requirements associated with these rights.

- Users may download and print one copy of any publication from the public portal for the purpose of private study or research.

- You may not further distribute the material or use it for any profit-making activity or commercial gain

- You may freely distribute the URL identifying the publication in the public portal 


\title{
Portable Vector Flow Imaging Compared with Spectral Doppler Ultrasonography
}

\author{
Tommaso Di Ianni, Kristoffer Lindskov Hansen, Carlos Armando Villagómez Hoyos, Ramin Moshavegh, \\ Michael Bachmann Nielsen, and Jørgen Arendt Jensen, IEEE Fellow
}

\begin{abstract}
In this study, a vector flow imaging (VFI) method developed for a portable ultrasound scanner was used for estimating peak velocity values and variation in beam-to-flow angle over the cardiac cycle in vivo on healthy volunteers. Peak-systolic velocity (PSV), end-diastolic velocity (EDV), and resistive index (RI) measured with VFI were compared to spectral Doppler ultrasonography (SDU). Seventeen healthy volunteers were scanned on the left and right common carotid arteries (CCAs). The standard deviation (SD) of VFI measurements averaged over the cardiac cycle was $7.3 \%$ for the magnitude and $3.84^{\circ}$ for the angle. Bland-Altman plots showed a positive bias for the PSV measured with SDU (mean difference: $0.31 \mathrm{~m} \mathrm{~s}^{-1}$ ), and Pearson correlation analysis showed a highly significant correlation $(r=0.6 ; p<0.001)$. A slightly positive bias was found for EDV and RI measured with SDU (mean difference: $0.08 \mathrm{~m} \mathrm{~s}^{-1}$ and $-0.01 \mathrm{~m} \mathrm{~s}^{-1}$, respectively). However, the correlation was low and not significant. The beam-to-flow angle was estimated over the systolic part of the cardiac cycle, and its variations were for all measurements larger than the precision of the angle estimation. The range spanned deviations from $-25.2^{\circ}(-6.0 \mathrm{SD})$ to $23.7^{\circ}$ (4.2 SD) with an average deviation from $-15.5^{\circ}$ to $9.7^{\circ}$. This can significantly affect PSV values measured by SDU as the beam-toflow angle is not constant and not aligned with the vessel surface. The study demonstrates that the proposed VFI method can be used in vivo for the measurement of PSV in the CCAs, and that angle variations across the cardiac cycle can lead to significant errors in SDU velocity estimates.
\end{abstract}

Index Terms-Vector flow imaging, Transverse oscillation, Synthetic aperture sequential beamforming, Portable ultrasound, Spectral Doppler ultrasonography

\section{INTRODUCTION}

Stroke is currently one of the leading causes of mortality worldwide and is responsible for long-term disability representing a substantial portion of the global healthcare cost. Although lifestyle adjustments have constantly reduced its

This work was supported by grant 82-2012-4 from the Danish National Advanced Technology Foundation and by BK Ultrasound, Herlev, Denmark. The authors wish to thank all the volunteers who participated in the study.

T. Di Ianni was with the Center for Fast Ultrasound Imaging, Department of Electrical Engineering, Technical University of Denmark, DK-2800 Kongens Lyngby, Denmark. He is now with the Department of Radiology, Molecular Imaging Program, Stanford University, Palo Alto, CA 94305, USA (e-mail: todiian@stanford.edu).

C. A. Villagómez Hoyos and R. Moshavegh were with the Center for Fast Ultrasound Imaging, Department of Electrical Engineering, Technical University of Denmark, DK-2800 Kongens Lyngby, Denmark. They are now with BK Ultrasound, DK-2730 Herlev, Denmark.

K. L. Hansen and M. B. Nielsen are with Department of Diagnostic Radiology, Rigshospitalet, Copenhagen University Hospital, DK-2100 Copenhagen, Denmark.

J. A. Jensen is with the Center for Fast Ultrasound Imaging, Department of Electrical Engineering, Technical University of Denmark, DK-2800 Kongens Lyngby, Denmark. incidence in the last years, the burden is expected to increase in the future due to increasing life expectancy [1]. Ischemic stroke caused by vessel occlusion following atherosclerotic disease accounts for $80 \%$ of cerebrovascular events, and an early intervention by carotid endarterectomy or stenting has proven beneficial in patients with severe carotid stenosis, i.e. $\geq 70 \%$ narrowing [2]-[4]. On the contrary, surgical treatment is not recommended among patients with mild or moderate stenosis [3].

The measurement of peak-systolic velocity (PSV) via spectral Doppler ultrasonography (SDU) is widely accepted for the grading of stenosis in the carotid arteries (CAs) [5], [6]. Under the assumption that the velocity correlates with the degree of vessel narrowing, PSV can be used to discriminate which patients must undergo surgical or medical treatment through a non-invasive and risk-free procedure. In particular, SDU examination is recommended in presence of severe stenosis, where artifacts and aberrations make it challenging to grade the disease using B-mode images alone [6].

Despite continuous technological improvements, SDU measurements are still affected by several limitations [5]. First, the velocity magnitude can be only quantified in a single location or a limited number of locations along the probing beam. Second, the velocity estimation is limited to the component parallel to the beam direction, and the operator is normally required to manually compensate for the beam-to-flow angle. The identification of the flow angle can be cumbersome, in particular in the presence of vessels with severe and morphologically complex stenosis. In addition, the angle can be expected to change over the cardiac cycle. As a consequence, the accuracy and precision of SDU are prone to system- and operator-dependent errors that impair the role of diagnostic ultrasound as a reliable tool for the grading of CA stenosis [7]. Several studies have shown that varying insonation angles in SDU measurements provide considerable differences in PSV resulting, in many cases, in uncertain grading of the stenosis [7], [8]. The issue is worsened by the lack of a broadly accepted consensus on whether an insonation angle equal to or $\leq 60^{\circ}$ must be used for the measurement of PSVs [7].

Vector flow imaging (VFI) estimates both the velocity magnitude and angle and eliminates the need for manual angle adjustments, therefore potentially improving the reliability of quantitative velocity measurements [9]. Several VFI methods have been proposed based on multi-beam approaches [10], [11], speckle tracking [12], and transverse oscillations (TOs) [13], [14]. The estimation of velocity vectors was also combined with parallel acquisition techniques for synthetic 
aperture (SA) and plane wave (PW) imaging to achieve high frame rates and a high precision [15]-[18].

Furthermore, the estimated magnitude and angle were used to correct and improve the Doppler spectrum calculation [19], [20]. A comprehensive review of VFI methods and applications can be found in [21]-[23].

A number of studies have been published aiming to validate the VFI velocities in the CAs in vivo. Hansen et al. [24] investigated the equivalence between three VFI implementations against magnetic resonance angiography (MRA). Pedersen et al. [25] compared PSV, end-diastolic velocity (EDV), resistive index (RI), and flow angle obtained by VFI implemented on a commercial platform with SDU measurements. Tortoli et al. [26] measured the PSV in healthy volunteers and patients with CA stenosis using two VFI methods and performed a comparison with SDU. The two methods were based on an angle tracking approach [27] and PW vector Doppler [28]. Recently, Jensen et al. investigated the accuracy and precision of PW VFI by comparing measured velocities with computational fluid dynamic simulations and MRA measurements [29]. A recent study using a convex array comparing VFI and SDU for the portal vein in the liver scanned in subcostal and intercostal views showed that VFI results were consistent in the two views but SDU was not, indicating that a poor beamto-flow angle in SDU can yield inconsistent results [9].

Alongside the support for high-frame-rate imaging, parallel techniques have the advantage of providing continuous data acquisition, which makes the velocity field available at any time in the entire image. However, PW and SA implementations have very high demands in terms of calculations per second and data rates as a full image has to beamformed for each emission using all transducer elements. This has so far precluded real time implementations of SA and PW vector flow on commercial ultrasound systems. A 2-D VFI method was recently proposed for a portable ultrasound system based on a hand-held probe for the acquisition of the data connected via wireless or USB to an external mobile device, where the processing is performed [30]. The method combines SA sequential beamforming (SASB) [31] and directional TO [32] to lower the data rate and computational requirements. For this approach a simple, static first stage beamformer sums all the element signals to one signal for each emission. This reduces the data rate and processing demands by a factor equal to the number of active elements, which is between 64 to 192, while retaining the advantages of a SA acquisition sequence.

The objective of the current study is to investigate the beamto-flow angle variation across the cardiac cycle and its influence on SDU estimates, and to validate the imaging sequence developed in [30]. The evaluation is performed in vivo on healthy volunteers, and compares quantitative velocity metrics measured with both VFI and SDU. Seventeen volunteers were scanned on the left and right common carotid artery (CCA) for a total of 34 datasets. The precision of VFI in the detection of beam-to-flow angle, PSV, and EDV was evaluated. PSV, EDV, and RI measurements using VFI and SDU were compared to examine the presence of a statistically significant correlation between the two methods, and the variation of the beam-toflow angle over the cardiac cycle was estimated along with its influence on current SDU measurements.

\section{MATERIALS AND METHODS}

\section{A. Data acquisition}

Seventeen healthy volunteers entered the study (13 males and 4 females; age: 24-43 years; mean age: 30.2 years). The study was approved by The Danish National Committee on Biomedical Research Ethics (Approval number: (KF)07307579). Both the left and right CCAs were scanned after the volunteer had been resting for approximately $10 \mathrm{~min}$ to ensure stationary flow conditions. The scans were performed by an experienced radiologist (KLH) approximately $2 \mathrm{~cm}$ below the carotid bifurcation in a longitudinal view. For each CCA, the measurements with SDU and VFI were performed in sequence.

A commercial ultrasound scanner (BK5000; BK Ultrasound, Herlev, Denmark) was used with a $4.1-\mathrm{MHz}$ linear array transducer (Linear Array 8L2; BK Ultrasound) for the SDU measurement. A range gate was positioned at the center of the vessel, and the beam direction and scan settings were tuned for an optimal spectral velocity estimation [33]. A cursor was moved parallel to the vessel wall for angle compensation. In Fig. 1, an example of the duplex view is shown for one volunteer. The PSV, EDV, and RI were recorded from the scanner for each volunteer. In this system, quantitative metrics are calculated from $8 \mathrm{~s}$ of SDU data. No additional information was provided by the manufacturer regarding the estimation of quantitative metrics.

For the VFI measurement, the same transducer was connected to the SARUS scanner [34]. The acquisition was performed using the sequence described in [30], consisting of six flow emissions interleaved with one B-mode emission. The pulse repetition frequency (PRF) was equal to $15 \mathrm{kHz}$, giving an effective PRF of $15 /(6+1)=2.1 \mathrm{kHz}$. The intensities and mechanical index (MI) of the sequence were measured prior to the scan session. The MI was equal to 0.91 and the derated $I_{\text {spta }}$ was $305.74 \mathrm{~mW} \mathrm{~cm}^{-2}$, in compliance with the US Food and Drug Administration regulations [35], [36]. The vessel was identified using a preview in MATLAB (The MathWorks, Inc., Natick, MA, USA), and $8 \mathrm{~s}$ of element data were subsequently stored. The preview was not available during the acquisition of the data. The VFI measurement was carried out right after the SDU with the volunteer kept in resting position.

\section{B. Processing}

The processing of the VFI data was performed off-line in MATLAB, and the high-resolution images (HRIs) were beamformed using the BFT3 toolbox [37]. A dual-stage clutter filter consisting of a moving average subtraction and an energybased filter was applied to remove the tissue signal from the flow data as described in [30], [38]. The energy based filter was selected due to its improved ability to separate the stationary component from the flow component as demonstrated in [29], [38]. The lateral and axial velocities were estimated using 16 HRIs by means of a 2-D autocorrelation estimator [32], and the velocity ranges were shifted to fit the expected velocity values and limit the influence of aliasing [30]. Finally, a median filter 


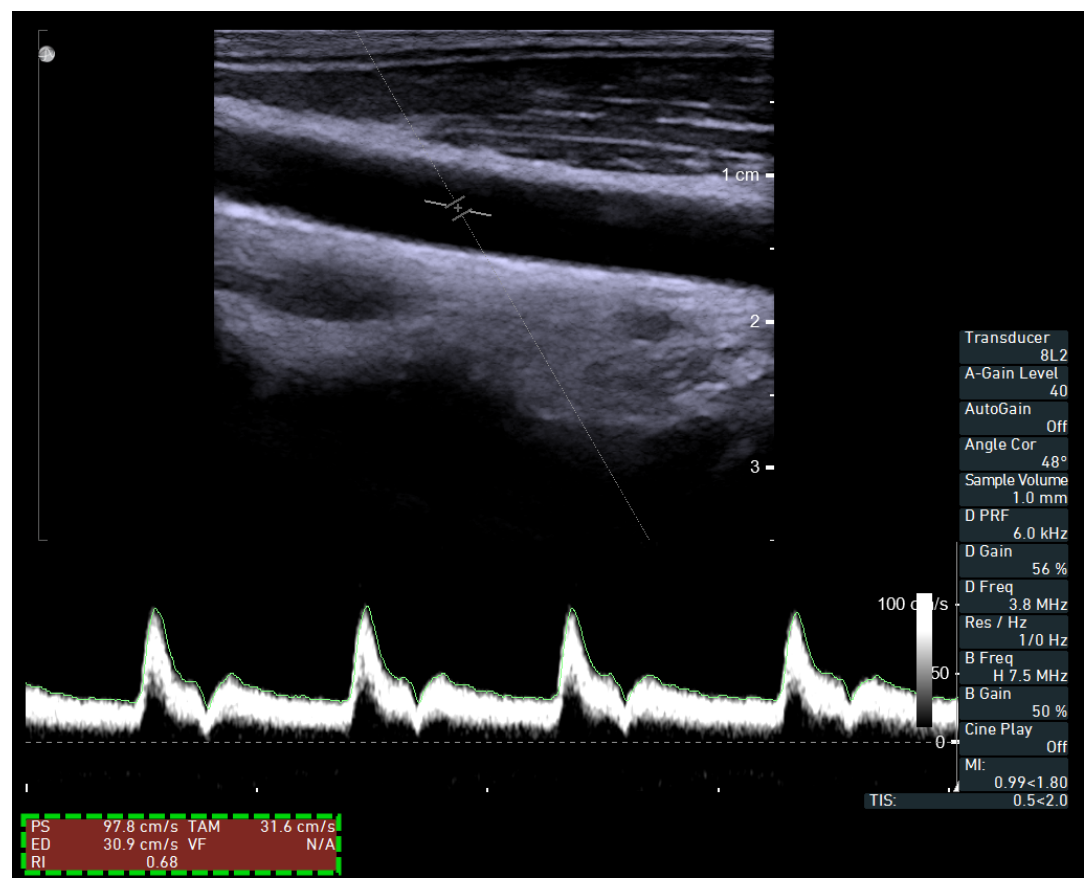

Fig. 1. Duplex view on the BK5000 scanner used for SDU measurements. The range gate was positioned at the center of the vessel, and the scanning settings were optimized for the spectral velocity estimation. The PSV, EDV, and RI are calculated from $8 \mathrm{~s}$ of SDU data in this system and are reported in the box highlighted by the green dashed line.

was applied within a temporal window of $10 \mathrm{~ms}$ and a spatial window of $0.5 \times 0.5 \mathrm{~mm}^{2}$.

The processed VFI and B-mode images were displayed overlaid using a visualization tool developed in-house in MATLAB. The velocity magnitude and angle were encoded using a color wheel as shown in Fig. 2. The frame rate was 300 frames/s for the VFI and 33 frames/s for the B-mode images. The video sequence was paused during the peak systolic phase to obtain the best possible indication of the vessel extent, and a region of interest (ROI) was placed by a radiologist (KLH) at the center of the lumen resembling the dimensions of the SDU range gate (blue box in Fig. 2). This operation was performed blinded to the corresponding result of the SDU measurement.

\section{VFI performance evaluation}

The velocity magnitudes and angles inside the selected ROI were used for the performance evaluation and for the estimation of quantitative metrics. A 2-D median filter was first applied spatially over the ROI obtaining magnitude and angle waveforms as a function of the time. Example waveforms for data set 3 are shown in Fig. 3, where the median magnitude into the ROI is displayed in the top plot and the median angle is displayed in the bottom for $8 \mathrm{~s}$ of VFI data. The magnitude peaks (red circles) were identified using an automatic MATLAB routine, and the mean PSV value and standard deviation (SD) were calculated from $8 \mathrm{~s}$ of data. The position of the peaks was used for the identification of the end-diastolic phase, defined as the interval between $80 \%$ and $90 \%$ of the cardiac cycle. The magnitude values considered for the mean EDV calculation are displayed in red in the top plot of Fig. 3. The RI was calculated from the mean detected PSV and EDV as (PSV-EDV)/PSV.

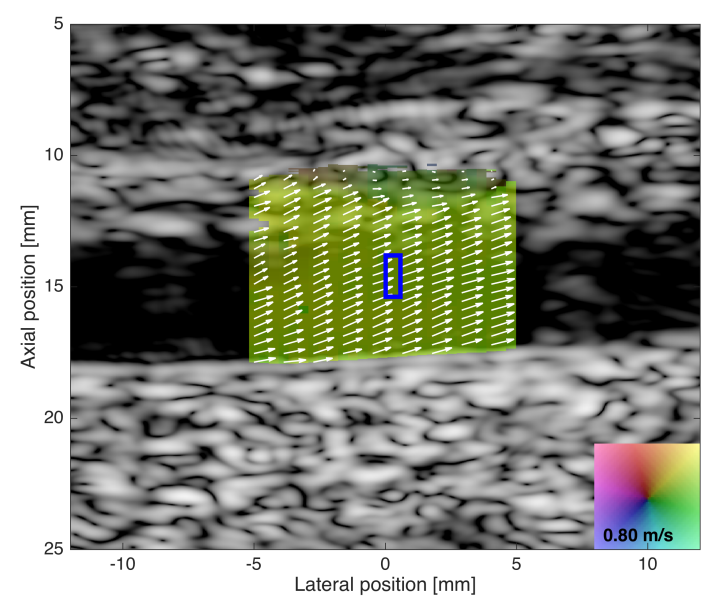

Fig. 2. Example of visualization for the VFI measurements. The processed VFI and B-mode images were displayed overlaid. The velocity magnitude and angle are encoded using the color wheel in the bottom-right corner, and the arrows show the local velocity vectors. A ROI (blue box) was placed by a radiologist at the center of the lumen resembling the dimensions of the corresponding SDU range gate.

\section{Mean profiles and estimation of angles}

Mean profiles were calculated from the magnitude and angle waveforms in Fig. 4. The peak positions of the autocorrelation functions were used to find the mean cardiac cycles in the $8 \mathrm{~s}$ of VFI data. The waveforms for the individual beats were then aligned through a cross-correlation to eliminate beat-tobeat variations. The mean and SD were calculated from the aligned waveforms and are displayed in Fig. 4 for the same volunteer. The top plot shows the mean magnitude profile in 

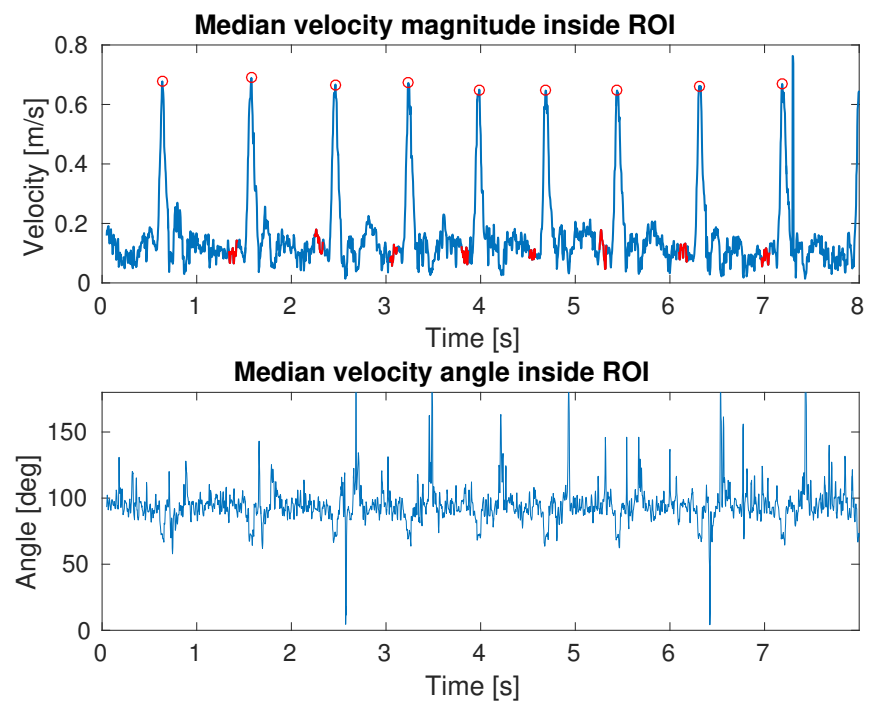

Fig. 3. Median velocity magnitude (top) and angle (bottom) into the ROI for data set 3 . The magnitude peaks (red circles) were identified using a MATLAB routine, and the mean PSV value and SD were calculated from $8 \mathrm{~s}$ of VFI data. The end-diastolic phase was defined as the interval between $80 \%$ and $90 \%$ of the cardiac cycle. The magnitude values used in the calculation of the mean EDV and SD are displayed in red. The velocity angle for $8 \mathrm{~s}$ of VFI data are shown in the bottom plot.

red and the SD as the shadowed gray region. For this volunteer, the relative SD averaged over the duration of the cardiac cycle was $4.43 \%$.

The corresponding angle estimates are shown below with the same time axis. The mean angle across the whole cardiac cycles is $93.95^{\circ}$, range $69.22^{\circ}$ to $134.48^{\circ}$, precision $=8.43^{\circ}$ $\left(4.68 \%\right.$ relative to $\left.180^{\circ}\right)$. The velocities around 0.4 seconds are low at the on-set of the diastolic phase. At such high angles the axial velocity is low, and the echo canceling removes most of the signal energy, thus, making the axial velocity estimation inaccurate in the diastolic phase. This is reflected in the large spikes for the angle estimates, which are considered outliers. Consistent angle estimates with few outliers can be found in the systolic phase. Defining it as the first $30 \%$ of the full cardiac cycle gives an angle span from $69.22^{\circ}$ to $104.78^{\circ}$ with a precision of $7.47^{\circ}(4.15 \%)$.

\section{E. Statistical analysis}

The measured quantities from $n=34$ CCAs were considered independent in the statistical analysis. The PSV, EDV, and RI measured with VFI and SDU were compared using Bland-Altman plots to evaluate the differences between the two methods. The mean difference and the $95 \%$ limits of agreement (LOA), defined as the mean $\pm 1.96 \mathrm{SD}$ of the difference, were reported.

The presence of a statistically significant linear relationship between pairs of variables measured with the two methods was examined by using a Pearson bivariate correlation analysis. The strength of the correlation was evaluated with the correlation coefficient $r$, and a $p$-value $\leq 0.05$ was considered statistically significant. The statistical analysis was performed in RStudio 1.1.442.

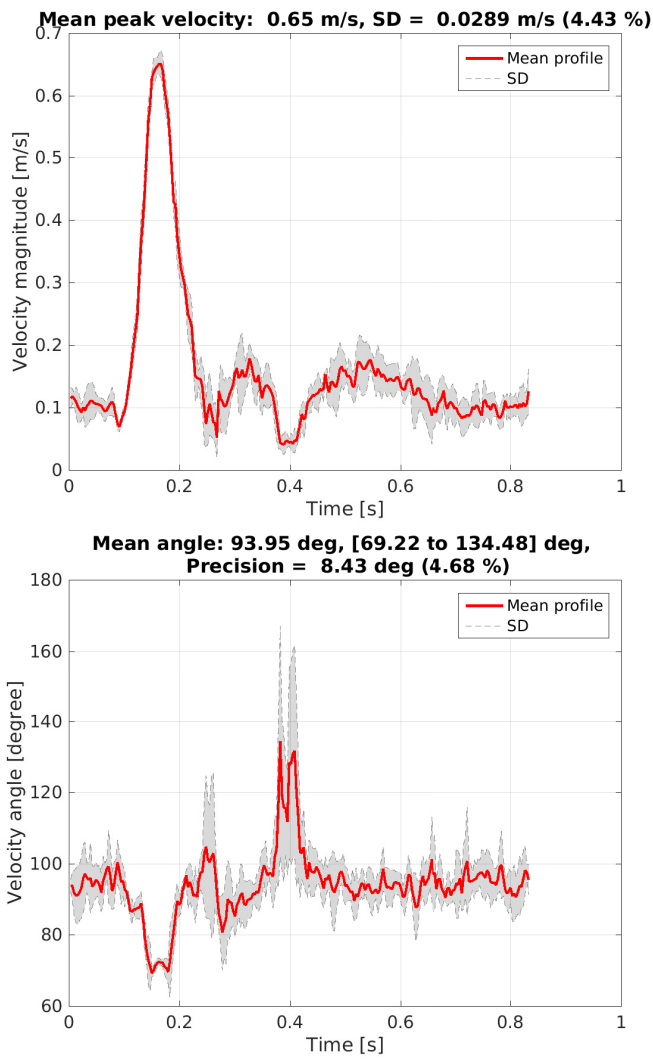

Fig. 4. Aligned velocity profiles for data set 3 (top). The red curve is the mean velocity magnitude and the gray shaded area is one SD. The lower graph shows the corresponding angle estimates with the red curve showing the mean angle.

\section{RESULTS}

\section{A. VFI performance evaluation}

The PSV and EDV estimated from the 34 measured CCAs are reported in Fig. 5. The red squares and blue asterisks show the estimated PSV and EDV, respectively, and the whiskers show the SD. The averaged SD was $0.027 \mathrm{~m} \mathrm{~s}^{-1}$ for PSV and $0.030 \mathrm{~m} \mathrm{~s}^{-1}$ for EDV.

\section{B. Comparison between VFI and SDU}

A box-and-whisker plot of PSV and EDV measured with VFI and SDU is displayed in Fig. 6. Each box spans the first quartile to the third quartile, the segment inside the rectangle shows the median, and the whisker shows the minimum and maximum measured values. The mean $\pm \mathrm{SD}$ are reported in Table I for the measured PSV, EDV, and RI.

Fig. 7 shows the scatter plots for PSV (a) and EDV (b) measured with SDU and VFI. The solid line displays the linear regression, and the shadowed area is the $95 \%$ confidence interval. Results from the Pearson correlation analysis are reported in Fig. 7 and summarized in Table II.

Fig. 8 shows the Bland-Altman plots for PSV, EDV, and RI measured with the two methods. The mean difference is displayed as a dashed line, and the continuous lines show the 95\% LOA. The results of the Bland-Altman analysis are summarized in Table III. 


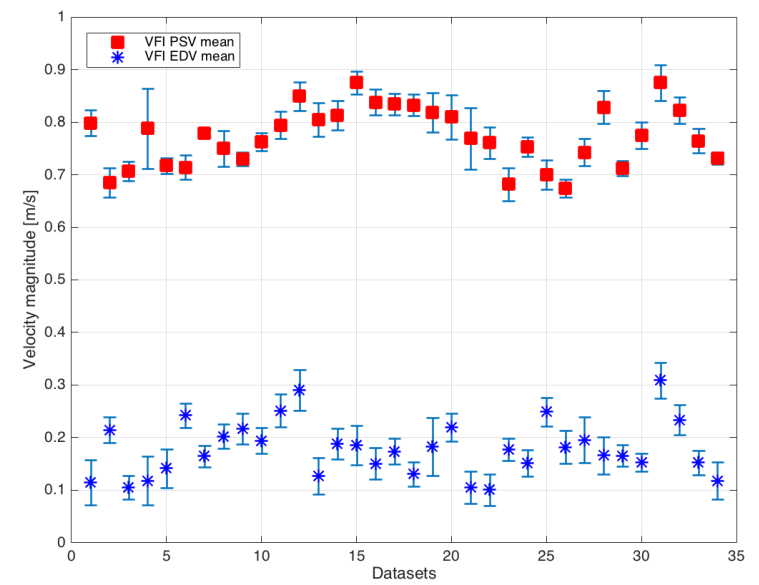

Fig. 5. PSV and EDV estimated from $n=34$ measured CCAs. The red squares are the PSVs and the blue asterisks the EDVs estimated from $8 \mathrm{~s}$ of VFI data. The whiskers show the SD with an average value of $0.027 \mathrm{~m} \mathrm{~s}^{-1}$ for the PSV and $0.030 \mathrm{~m} \mathrm{~s}^{-1}$ for the EDV.

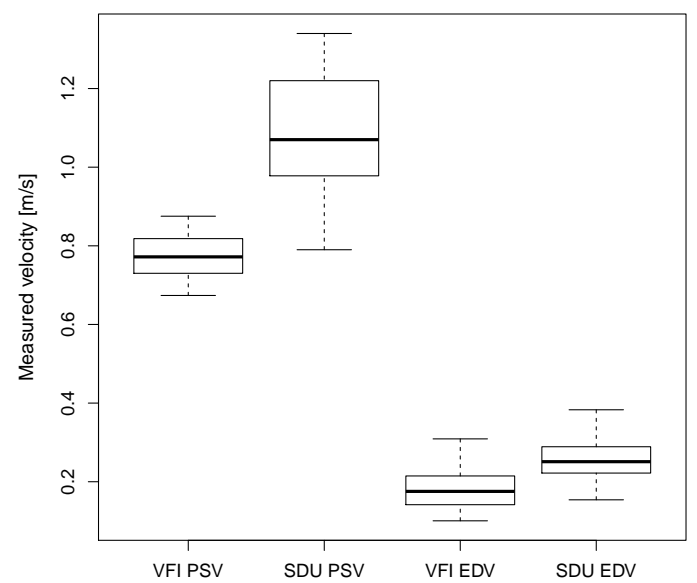

Fig. 6. Box-and-whisker plot of PSV and EDV measured with VFI and SDU. Each box spans the first to the third quartile, the segment shows the median, and the whisker shows the minimum and maximum measured values $(n=34)$.

TABLE I

SUMMARY OF MEASURED PSV, EDV, AND RI (MEAN \pm SD)

\begin{tabular}{lccc}
\hline & $\begin{array}{c}\text { PSV } \\
{\left[\mathrm{m} \mathrm{s}^{-1}\right]}\end{array}$ & $\begin{array}{c}\text { EDV } \\
{\left[\mathrm{m} \mathrm{s}^{-1}\right]}\end{array}$ & RI \\
\hline SDU & $1.08 \pm 0.16$ & $0.26 \pm 0.05$ & $0.76 \pm 0.06$ \\
VFI & $0.77 \pm 0.06$ & $0.18 \pm 0.05$ & $0.77 \pm 0.07$ \\
\hline
\end{tabular}

TABLE II

PEARSON'S CORRELATION ANALYSIS

\begin{tabular}{c|c|c|c}
\hline & PSV & EDV & RI \\
\hline$r$ & 0.60 & 0.29 & 0.23 \\
$p$ & $\leq 0.001$ & 0.097 & 0.2 \\
\hline
\end{tabular}

TABLE III

BLAND-ALTMAN ANALYSIS SUMMARY

\begin{tabular}{l|c|c|c}
\hline & $\begin{array}{c}\text { PSV } \\
{\left[\mathrm{m} \mathrm{s}^{-1}\right]}\end{array}$ & $\begin{array}{c}\text { EDV } \\
{\left[\mathrm{m} \mathrm{s}^{-1}\right]}\end{array}$ & RI \\
\hline Avg. difference & 0.31 & 0.08 & -0.01 \\
LOA & {$[0.05,0.57]$} & {$[-0.04,0.19]$} & {$[-0.16,0.14]$} \\
\hline
\end{tabular}

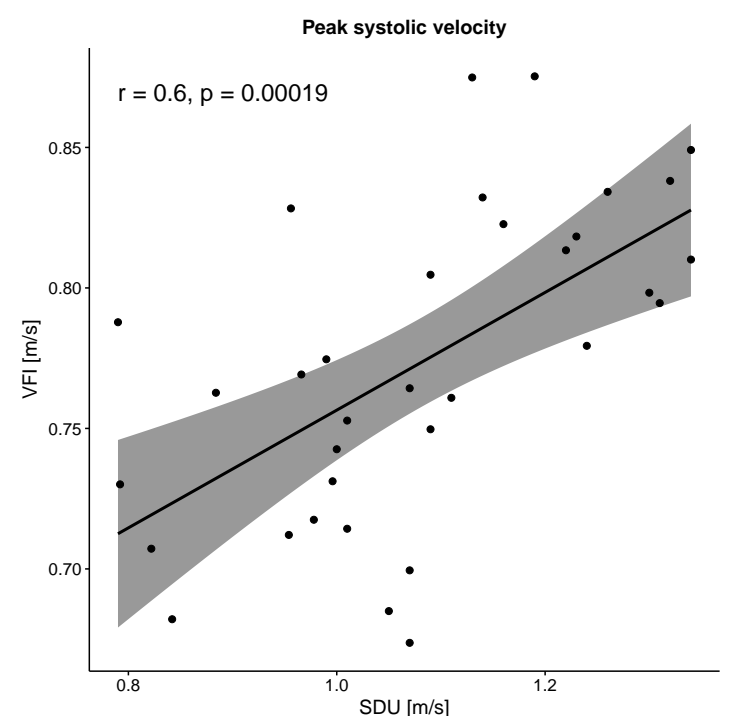

(a)

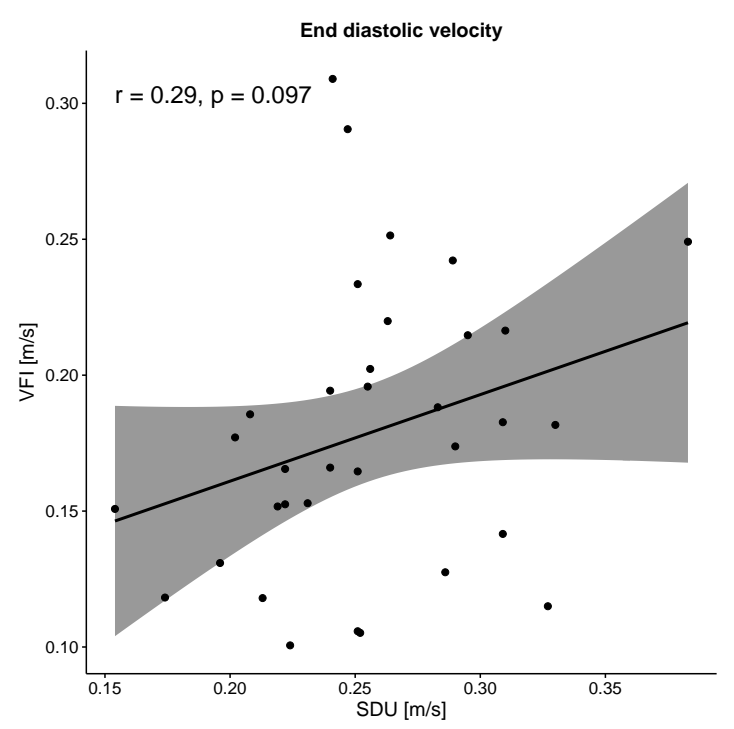

(b)

Fig. 7. Scatter plots of PSV (a) and EDV (b) measured with SDU and VFI The solid line represents the linear regression, and the grey shadowed area is the 95\% confidence interval. The correlation coefficient $r$ and $p$-value from the Pearson correlation analysis are reported on the upper-left corner of the plots $(n=34)$.

\section{Angle variation}

The angle variation across the cardiac cycle was estimated for all data sets. A typical example for data set number 3 is shown in Fig. 4. The red curve in the bottom graph is the mean angle and the gray shaded area indicates one SD (the precision). There is a clear change in angle of $-24.7^{\circ}$ during the systolic acceleration phase. This is 3.3 times the angle SD of $7.47^{\circ}$. The angle estimates are, thus, deviating from the main direction in the cardiac cycle and could indicate flow slightly moving downwards towards the vessel boundary.

The angle deviation in the systolic phase was calculated for all data sets and is shown in Fig. 9, where the mean angle across the cardiac cycle has been subtracted and the deviation from this is plotted. The red vertical curves show the precision 

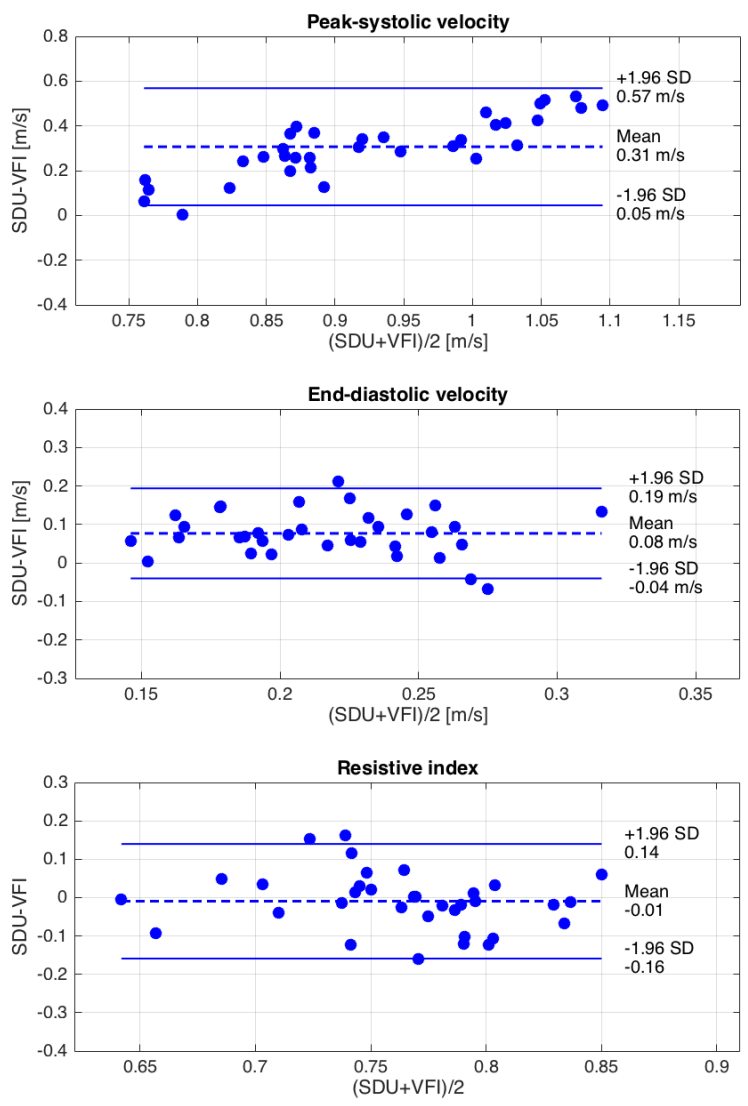

Fig. 8. Bland-Altman plots for the PSV (top), EDV (middle), and RI (bottom) measured with SDU and VFI. For each variable, the plotted dots are relative to the same CA measured with the two methods. The mean difference is reported as an horizontal dashed line, and the LOA are shown as continuous lines.

for the estimated angle as $\pm 1 \mathrm{SD}$. The green curves are the $\mathrm{SD}$ of the mean curve found by averaging across all heart beats. This SD is calculated by dividing the variance of the angle estimates by the number of cardiac cycles and is the estimated SD of the mean curve for all the aligned cardiac cycles. The maximum and minimum detected angles for the mean angle curve are show as the blue circles and red squares. This shows the largest angle deviation in the mean curve for the systolic part of the cardiac cycle. Across all data sets the averaged SD is $5.23^{\circ}$ with a range from $3.49^{\circ}$ to $7.47^{\circ}$. The corresponding overall SD of the mean angle curves is $1.98^{\circ}$.

\section{DISCUSSION}

In this study, a 2-D VFI method developed for a portable ultrasound scanner was investigated in vivo, and a comparison study was performed against SDU to reveal whether the two methods are statistically equivalent with respect to the detection of quantitative flow metrics and to evaluate the influence from the beam-to-flow angle. Average SDs of $4.43 \%$ and $7.47^{\circ}$ were calculated for the mean magnitude and angle profiles in Fig. 4, demonstrating consistency of the detected velocities over the acquisition time. The performance of VFI in the detection of PSV and EDV was evaluated, with an average SD of $0.027 \mathrm{~m} \mathrm{~s}^{-1}$ for PSV and $0.030 \mathrm{~m} \mathrm{~s}^{-1}$ for EDV.
PSVs measured with SDU and VFI showed a highly significant correlation $(r=0.6, p \leq 0.001)$.

In Fig. 5, a higher SD was measured for CCAs number 4 and 21, where clutter filtering faults caused errors in the velocity estimation. The energy-based clutter filter employed in this study (2nd stage), although necessary for an effective attenuation of the clutter from the moving tissue, relies upon an energy threshold [38]. The selection of the threshold is critical to the performance of the estimator and can bias the estimated velocities, as discussed in [30]. A single threshold was used for the 34 CCAs, even though varying signal amplitudes can be expected from a broad population. Further research is needed to determine the energy threshold in an adaptive way, as highlighted in [38].

The Bland-Altman analysis for the PSV in Fig. 8 showed a systematic bias of SDU with respect to VFI, with a mean difference of $0.31 \mathrm{~m} \mathrm{~s}^{-1}$. The result is in good agreement with what previously found in [26], where an overestimation of about $0.2 \mathrm{~m} \mathrm{~s}^{-1}$ was observed with lower average velocities compared with those detected in the current study $\left(0.806 \mathrm{~m} \mathrm{~s}^{-1}\right.$ against $1.08 \mathrm{~m} \mathrm{~s}^{-1}$ for SDU and $0.59 \mathrm{~m} \mathrm{~s}^{-1}$ against $0.77 \mathrm{~m} \mathrm{~s}^{-1}$ for VFI). A trend towards higher deviations for larger PSV values can also be seen in Fig. 8. This overestimation can partly be attributed to spectral broadening effects in SDU [39][41] and from deviations in angle (see below). Furthermore, in the commercial platform used in this study, quantitative measures are estimated from the envelope of the spectrum, displayed in Fig. 1 as a continuous green line. This is expected to further contribute to the positive bias of SDU. The two methods showed a highly significant correlation in the measurement of PSVs $(r=0.6, p \leq 0.001)$, conversely to what was previously found in [25]. The result is probably due to the improved temporal resolution, which allows for a better detection of the peaks in the magnitude profile.

A slightly positive bias was found for the SDU measurement of EDVs compared with VFI, with a mean difference of $0.08 \mathrm{~m} \mathrm{~s}^{-1}$. However, the two methods showed a poor correlation and no statistical significance $(r=0.29 ; p>0.05)$. The EDV is also the most difficult velocity measure to estimate due to the low velocity, and, hence influence from the echo canceling filters, which removes much of the energy at low velocities. Furthermore, it is unknown how the EDV is estimated in the commercial scanner, therefore it is difficult to compare the two measurements. A negligible bias was found for the RI, and the study showed a low correlation between SDU and VFI with no statistical significance $(r=0.23 ; p>0.05)$. This is probably due to the bias of both PSV and EDV affecting the RI value.

The VFI estimated beam-to-flow angle and its variation in systole has also been investigated. Fig. 9 shows that for each data set either the minimum or maximum angle is close to one $\mathrm{SD}$ of the estimates. This is when either the blue circle or the red square is near the value of the red vertical bar for each data set. The opposite value for the maximum or minimum angle is significantly larger than the SD by a factor of 2 to 4 . This indicates a motion away from the mean angle in a direction either towards the transducer or away from it. The flow, thus, seems to deviate from the vessels center axis at peak velocities 


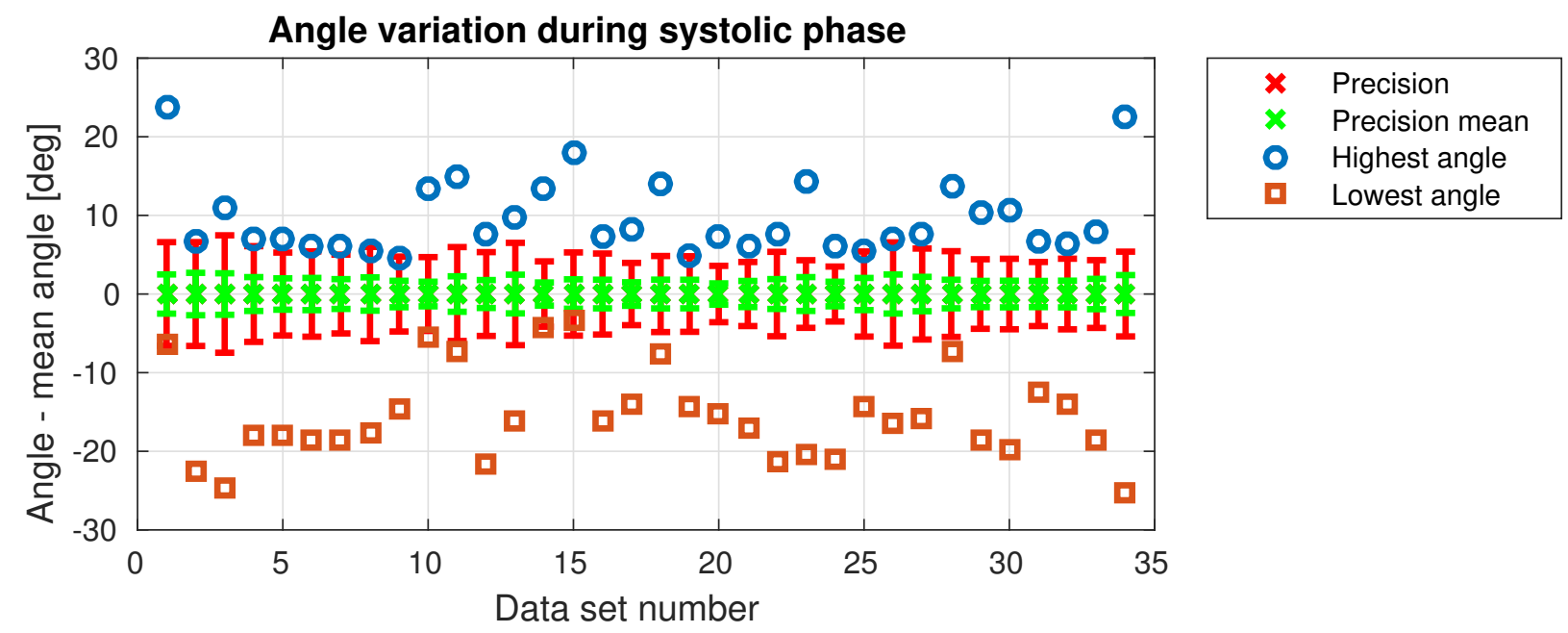

Fig. 9. The angle deviation from the mean beam-to-flow angle for all data sets for the systolic part of the cardiac cycles (first $30 \%$ of the cardiac cycle). The red vertical curves show the estimated SD of the angle and the green curves is the precision of the mean angle curve across all heart beats for the individual data sets. The blue circles show the largest detected angles for the mean angle curves and the red squares are the minimum angles.

depending on whether the ROI is below or above the center axis. These deviations are between $10^{\circ}$ to $25^{\circ}$ and indicate that the angle during peak systole does not follow the vessel boundary, but rather deviates from it.

This deviation can be due to physiological angle variations in different phases of the cardiac cycle. A similar angle variation can be expected for the SDU measurement. It is worth noticing that an an error of $\pm 5^{\circ}$ at an insonation angle of $60^{\circ}$ gives a velocity error of approximately $30 \%$ in the SDU measurement.

Finding the peak velocity in the cardiac cycle demands a good angle determination, as this directly scales the velocity found. In current commercial scanners the angle is aligned with the vessel wall, but during the peak acceleration phase the flow angle seems to change due to the expansion of the vessel also seen in the B-mode image.

The beam-to-flow angle could therefore be incorrect for SDU. Having a range gate covering the central part of the vessel will worsen the problem as the flow deviation pointing towards the vessel boundary will make the actual angle smaller and thereby increase the axial velocity component measured in SDU. The peak velocity would be overestimated, which is also consistently seen for the results here. Furthermore there is no method to consistently compensate for this, as the true angle deviation cannot be known in SDU, but can readily be found in VFI. The wrong angle correction will also affect RI through the PSV.

The angle variation across the cardiac cycle has been used for calculating the likely range of velocities from SDU, when the angle spread is included. The angle variation across the systolic phase estimated by VFI has been used for compensating the angle used in SDU. The spread in angle during systole was added and subtracted from the SDU angle, and this yields a range of possible PSV estimates. This is shown in Fig. 10, where the overestimation from spectral broadening has been compensated for by scaling the SDU PSV values to $90 \%$ of the estimated value. The red vertical lines indicates the possible

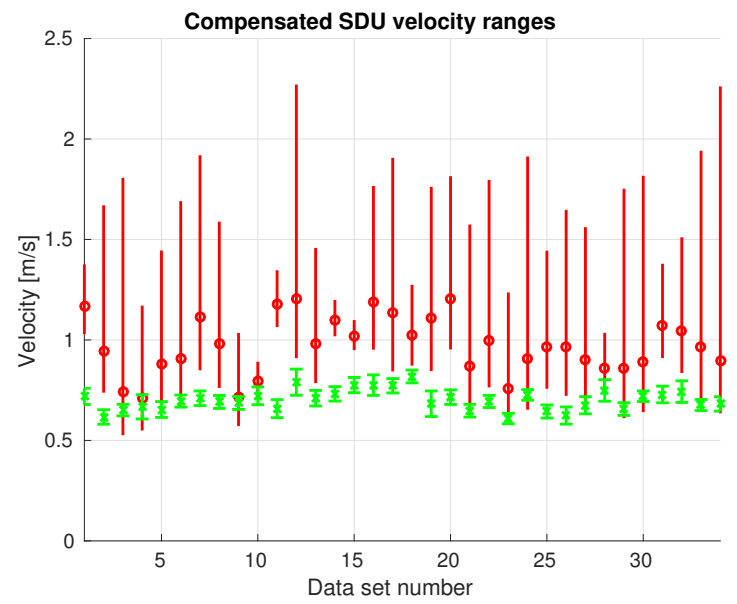

Fig. 10. Possible ranges for PSV SDU values corrected by the angle span detected from the VFI measurements. The red vertical curves shows the range of SDU PSV values and the green curves are the corresponding VFI PSV values.

PSV range, and the green vertical curves are the ranges for the VFI peak velocities. There is not full consistence between SDU and VFI but in 15 cases the possible SDU PSV values overlap the VFI estimates.

The accuracy and precision of SDU are significantly influenced by the fact that a single value is used for angle correction [7], [9]. Several studies have shown that varying insonation angles in SDU measurements provide considerable differences in PSV, resulting, in many cases, in uncertain grading of the stenosis [7], [8]. It was shown in [9], when estimating peak velocities in the portal vein, that inconsistent results were found for two different views for SDU, whereas results were consistent for VFI. The issue is worsened by the lack of a broadly accepted consensus on whether an insonation angle equal to $60^{\circ}$ or $\leq 60^{\circ}$ must be used for the measurement of PSVs [7].

The results shown here indicates a rather large angle vari- 
ation during the systolic phase with deviations in the range of $20^{\circ}$ to $30^{\circ}$. Compensating SDU PSV estimates with this brings some VFI and SDU PSV estimates in agreement. These angle estimates are, however, also affected by a number of factors in the VFI set-up. The axial velocity estimator used has a limited velocity range due to both the reduced $f_{p r f}$ from the length of the SASB sequence and the coupling with the lateral velocity component [42]. This made it necessary to compensate the velocity range for the axial velocities, as it exceeded the aliasing limit during peak systole. The high resolution data for velocity estimation are also summed over 6 emissions, and this will, at high velocities, de-correlate the data, so the summation is out of phase resulting in higher side lobes [43]. This increases the influence from the vessel wall signal, which can bias the velocity estimate, if the echo canceling does not full suppress tissue components.

The velocity range compensation for high velocities can be avoided by using a newly introduced interleaving scheme for SA flow imaging [44]-[46]. Here the individual emissions are repeated, and the effective pulse repetition frequency $f_{p r f, e f f}=f_{p r f} / N$, where $N$ is the number of emissions in the sequence, is replaced by $f_{p r f}$. This effectively increase the maximum detectable velocity by a factor of $N$, which is significant for patients with stenosis typically having peak velocities of $2-4 \mathrm{~m} / \mathrm{s}$.

The VFI method allows for the detection of quantitative metrics without any manual angle adjustments while significantly alleviating the burden of SA imaging sequences in terms of data rates and computational requirements. The VFI approach presented here uses a dual-stage beamforming and a relatively inexpensive velocity estimator based on directional TO. The processing demands are a factor of 64 to 192 times lower than for full implementations of SA flow imaging [47], [48]. Further, data rates as low as $14 \mathrm{MB} / \mathrm{s}$ were proven sufficient for achieving real time imaging performance using an emulated wireless probe and a commercially available tablet, where the processing was carried out with a frame rate of 26 frames/s [49]. However, the sequence can reach a maximum frame rate of 2140 frames/s, enabling the visualization of complex hemodynamic patterns like the formation of vortices in the internal CAs [50]-[52]. In addition, the 2-D VFI method provides quantitative velocities in the entire image continuously [50], enabling the possibility of retrospective quantitative measurements [16], [18], and it has the potential to solve the problem of sample volume placement in SDU measurements [53]. The main drawback of SASB VFI compared to the full data acquisition for SA or plane wave VFI in e.g. [29], [50], [51] is the higher side-lobes, which results in an increased standard deviation for the estimates. Also the field-of-view is more restricted, as the beams have to overlap to yield a functional transverse oscillation.

The main limiting factor of this study was the low number of volunteers. Furthermore, only healthy subjects were considered, and higher velocities proper of stenotic vessels were not tested. A third reference method like MRI could also be added, although this has a limited spatial and temporal resolution making precise comparisions difficult. In addition, no interor intra-observer studies were performed. The absence of a preview during the VFI acquisition made the measurements potentially affected by movements of the probe affecting the precise placement of the scan plan in the middle of the vessel, which could not be detected during the scan. It is worth pointing out that visual and acoustic feed-backs were used for the placement of the range gate during SDU measurements, while this was not available during the VFI acquisition on the research scanner. In addition, VFI and SDU measurements were not performed simultaneously.

\section{CONCLUSIONS}

The results of this study demonstrated that velocities estimated using a VFI approach developed for a portable ultrasound scanner can be used for the measurement of PSVs in the CCAs, alleviating the problems of sample placement and angle correction of SDU that hinder its reliability in the grading of CA stenosis. The overall precision of the velocity estimates was $5.6 \%$ across all measurement sets and the angle could be determined with an overall precision of $4.9^{\circ}$, which is significantly lower than the $20^{\circ}$ to $30^{\circ}$ angle variation estimated during the systolic phase. The VFI method, thus, offers a method for making quantitative estimates of the peak velocity in the carotid artery without angle correction. The study has also shown that a single, fixed angle correction with SDU can be dubious, as the angle probably is not constant during the cardiac cycle and does in general not follow the vessel wall at peak systole.

The method can be implemented on a commercial tablet and opens the possibility of spreading ultrasound imaging to a broader user population. Velocity profiles like the ones in Fig. 3 and Fig. 4 could be displayed in place of the SDU waveform. These profiles can be obtained everywhere in the VFI image, allowing for extended multi-gated measurements. The measurements can be conducted retrospectively, as all the velocity estimates are available continuously in the image, and the data rates and real-time processing demands are within the reach of a standard PC or tablet.

\section{REFERENCES}

[1] G. Donnan, M. Fisher, M. Macleod, and S. Davis, "Stroke," Lancet, vol. 371, no. 9624, pp. 1612-1623, 2008.

[2] North American Symptomatic Carotid Endarterectomy Trial Collaborators, "Beneficial effect of carotid endarterectomy in symptomatic patients with high-grade carotid stenosis," N. Engl. J. Med, vol. 325, no. 7, pp. 445-453, 1991 .

[3] H. Barnett, D. Taylor, M. Eliasziw, A. Fox, G. Ferguson, R. Haynes, R. Rankin, G. Clagett, V. Hachinski, D. Sackett, K. Thorpe, H. Meldrum, and J. Spence, "Benefit of carotid endarterectomy in patients with symptomatic moderate or severe stenosis," N. Engl. J. Med, vol. 339, no. 20, pp. 1415-1425, 1998.

[4] T. Brott, R. H. II, G. Howard, G. Roubin, W. Clark, W. Brooks, A. Mackey, M. Hill, P. Leimgruber, A. Sheffet, V. Howard, W. Moore, J. Voeks, L. Hopkins, D. Cutlip, D. Cohen, and J.J, "Stenting versus endarterectomy for treatment of carotid-artery stenosis," New England Journal of Medicine, vol. 363, no. 1, pp. 11-23, 2010.

[5] E. G. Grant, C. B. Benson, and G. L. M. et al, "Carotid artery stenosis: Gray-scale and Doppler US diagnosis - society of radiologists in ultrasound consensus conference," Radiology, vol. 229, no. 2, pp. 340-346, 2003.

[6] G. M. von Reutern, M. W. Goertler, N. M. Bornstein, M. D. Sette, D. H. Evans, A. Hetzel, M. Kaps, F. Perren, and et al., "Grading carotid stenosis using ultrasonic methods," Stroke, vol. 43, no. 3, pp. 916-921, 2012. 
[7] M. Tola and M. Yurdakul, "Effect of Doppler angle in diagnosis of internal carotid artery stenosis," J. Ultrasound Med., vol. 25, pp. 11871192, 2006.

[8] K. Logason, T. Bärlin, M. Jonsson, A. Boström, H. Hårdemark, and S. Karacagil, "The importance of Doppler angle of insonation on differentiation between 50-69\% and 70-99\% carotid artery stenosis," Eur. J. Vasc. Endovasc. Surg., vol. 21, pp. 311-313, 2001.

[9] A. H. Brandt, R. Moshavegh, K. L. Hansen, L. Lonn, J. A. Jensen, and M. Nielsen, "Vector flow imaging compared with pulse wave Doppler for estimation of peak velocity in the portal vein," Ultrasound Med. Biol., vol. 44, no. 3, pp. 593-601, 2018.

[10] P. Peronneau, J.-P. Bournat, A. Bugnon, A. Barbet, and M. Xhaard, "Theoretical and practical aspects of pulsed doppler flowmetry real-time application to the measure of instantaneous velocity profiles in vitro and in vivo," in Cardiovascular applications of ultrasound, R. Reneman, Ed. North Holland Publishing,, 1974, pp. 66-84.

[11] B. Dunmire, K. W. Beach, K.-H. Labs., M. Plett, and D. E. Strandness, "Cross-beam vector Doppler ultrasound for angle independent velocity measurements," Ultrasound Med. Biol., vol. 26, pp. 1213-1235, 2000.

[12] G. E. Trahey, J. W. Allison, and O. T. von Ramm, "Angle independent ultrasonic detection of blood flow," IEEE Trans. Biomed. Eng., vol. BME-34, no. 12 , pp. 965-967, 1987.

[13] J. A. Jensen and P. Munk, "A new method for estimation of velocity vectors," IEEE Trans. Ultrason., Ferroelec., Freq. Contr., vol. 45, no. 3 , pp. 837-851, 1998

[14] M. E. Anderson, "Multi-dimensional velocity estimation with ultrasound using spatial quadrature," IEEE Trans. Ultrason., Ferroelec., Freq. Contr., vol. 45, pp. 852-861, 1998

[15] S. I. Nikolov and J. A. Jensen, "In-vivo synthetic aperture flow imaging in medical ultrasound," IEEE Trans. Ultrason., Ferroelec., Freq. Contr., vol. 50, no. 7, pp. 848-856, 2003.

[16] J. A. Jensen, S. Nikolov, K. L. Gammelmark, and M. H. Pedersen, "Synthetic aperture ultrasound imaging," Ultrasonics, vol. 44, pp. e5e15, 2006.

[17] J. Udesen, F. Gran, K. L. Hansen, J. A. Jensen, C. Thomsen, and M. B. Nielsen, "High frame-rate blood vector velocity imaging using plane waves: simulations and preliminary experiments," IEEE Trans. Ultrason., Ferroelec., Freq. Contr., vol. 55, no. 8, pp. 1729-1743, 2008

[18] J. Bercoff, G. Montaldo, T. Loupas, D. Savery, F. Meziere, M. Fink, and M. Tanter, "Ultrafast compound Doppler imaging: providing full blood flow characterization," IEEE Trans. Ultrason., Ferroelec., Freq. Contr., vol. 58, no. 1, pp. 134-147, January 2011.

[19] I. K. Ekroll, T. Dahl, H. Torp, and L. Løvstakken, "Combined vector velocity and spectral Doppler imaging for improved imaging of complex blood flow in the carotid arteries," Ultrasound Med. Biol., vol. 40, no. 7, pp. 1629-1640, 2014

[20] J. Avdal, L. Løvstakken, H. Torp, and I. K. Ekroll, "Combined 2-D vector velocity imaging and tracking doppler for improved vascular blood velocity quantification," IEEE Trans. Ultrason., Ferroelec., Freq. Contr., vol. 64, no. 12, pp. 1795-1804, 2017.

[21] J. A. Jensen, S. I. Nikolov, A. Yu, and D. Garcia, "Ultrasound vector flow imaging I: Sequential systems," IEEE Trans. Ultrason., Ferroelec., Freq. Contr., vol. 63, no. 11, pp. 1704-1721, 2016.

[22] _ - "Ultrasound vector flow imaging II: Parallel systems," IEEE Trans. Ultrason., Ferroelec., Freq. Contr., vol. 63, no. 11, pp. 1722-1732, 2016.

[23] K. L. Hansen, M. B. Nielsen, and J. A. Jensen, "Vector velocity estimation of blood flow - A new application in medical ultrasound," Ultrasound, vol. 25, no. 4, pp. 189-199, 2017.

[24] K. L. Hansen, J. Udesen, C. Thomsen, J. A. Jensen, and M. B. Nielsen, "In vivo validation of a blood vector velocity estimator with MR angiography," IEEE Trans. Ultrason., Ferroelec., Freq. Contr., vol. 56, no. 1, pp. 91-100, 2009.

[25] M. M. Pedersen, M. J. Pihl, P. Haugaard, J. M. Hansen, K. L. Hansen, M. B. Nielsen, and J. A. Jensen, "Comparison of real-time in vivo spectral and vector velocity estimation," Ultrasound Med. Biol., vol. 38, no. 1, pp. 145-151, 2012.

[26] P. Tortoli, M. Lenge, D. Righi, G. Ciuti, H. Liebgott, and S. Ricci, "Comparison of carotid artery blood velocity measurements by vector and standard Doppler approaches," Ultrasound Med. Biol., vol. 41, no. 5, pp. 1354-1362, 2015.

[27] P. Tortoli, A. Dallai, E. Boni, L. Francalanci, and S. Ricci, "An automatic angle tracking procedure for feasible vector Doppler blood velocity measurements," Ultrasound Med. Biol., vol. 36, no. 3, pp. 488-496, 2010.

[28] S. Ricci, L. Bassi, and P. Tortoli, "Real-time vector velocity assessment through multigate Doppler and plane waves," IEEE Trans. Ultrason., Ferroelec., Freq. Contr., vol. 61, no. 2, pp. 314-324, 2014.
[29] J. Jensen, C. A. V. Hoyos, M. S. Traberg, J. B. Olesen, B. G. Tomov R. Moshavegh, S. H. B. Stuart, C. Ewertsen, K. L. Hansen, C. E. Thomsen, M. B. Nielsen, and J. A. Jensen, "Accuracy and precision of a plane wave vector flow imaging method in the healthy carotid artery," Ultrasound in Medicine \& Biology, vol. 44, no. 8, pp. 1727-1741, 2018.

[30] T. Di Ianni, C. Villagomez-Hoyos, C. Ewertsen, T. Kjeldsen, J. Mosegaard, and J. A. Jensen, "A vector flow imaging method for portable ultrasound using synthetic aperture sequential beamforming," IEEE Trans. Ultrason., Ferroelec., Freq. Contr., vol. 64, no. 11, pp. 1655-1665, 2017.

[31] J. Kortbek, J. A. Jensen, and K. L. Gammelmark, "Sequential beamforming for synthetic aperture imaging," Ultrasonics, vol. 53, no. 1, pp. $1-16,2013$.

[32] J. A. Jensen, "Directional transverse oscillation vector flow estimation," IEEE Trans. Ultrason., Ferroelec., Freq. Contr., vol. 64, no. 8, pp. 1194 1204, 2017.

[33] H. R. Tahmasebpour, A. R. Buckley, P. L. Cooperberg, and C. H. Fix, "Sonographic examination of the carotid arteries," Radiographics, vol. 25 , no. 6, p. 1, 2005.

[34] J. A. Jensen, H. Holten-Lund, R. T. Nilsson, M. Hansen, U. D. Larsen, R. P. Domsten, B. G. Tomov, M. B. Stuart, S. I. Nikolov, M. J. Pihl, Y. Du, J. H. Rasmussen, and M. F. Rasmussen, "SARUS: A synthetic aperture real-time ultrasound system," IEEE Trans. Ultrason., Ferroelec., Freq. Contr., vol. 60, no. 9, pp. 1838-1852, 2013.

[35] FDA, "Information for manufacturers seeking marketing clearance of diagnostic ultrasound systems and transducers," Center for Devices and Radiological Health, United States Food and Drug Administration, Tech. Rep., 2008.

[36] J. A. Jensen, M. F. Rasmussen, M. J. Pihl, S. Holbek, C. A. VillagomezHoyos, D. P. Bradway, M. B. Stuart, and B. G. Tomov, "Safety assessment of advanced imaging sequences, I: Measurements," IEEE Trans. Ultrason., Ferroelec., Freq. Contr., vol. 63, no. 1, pp. 110-119, 2016.

[37] J. M. Hansen, M. C. Hemmsen, and J. A. Jensen, "An object-oriented multi-threaded software beamformation toolbox," in Proc. SPIE Med. Imag., vol. 7968, March 2011, pp. 79680Y-1-79680Y-9.

[38] C. A. Villagomez-Hoyos, J. Jensen, C. Ewertsen, K. L. Hansen, M. B. Nielsen, and J. A. Jensen, "Energy based clutter filtering for vector flow imaging," in Proc. IEEE Ultrason. Symp., 2017, pp. 1-4.

[39] V. L. Newhouse, P. J. Bendick, and L. W. Varner, "Analysis of transit time effects on Doppler flow measurement," IEEE Trans. Biomed. Eng., vol. BME-23, pp. 381-387, 1976.

[40] V. L. Newhouse, E. S. Furgason, G. F. Johnson, and D. A. Wolf, "The dependence of ultrasound Doppler bandwidth on beam geometry," IEEE Trans. Son. Ultrason., vol. SU-27, pp. 50-59, 1980.

[41] A. Steinman, J. Tavakkoli, J. Myers, R. Cobbold, and K. Johnston, "Sources of error in maximum velocity estimation using linear phasedarray Doppler systems with steady flow," Ultrasound Med. Biol., vol. 27, no. 5 , pp. $655-664,2001$

[42] J. A. Jensen, "A new estimator for vector velocity estimation," IEEE Trans. Ultrason., Ferroelec., Freq. Contr., vol. 48, no. 4, pp. 886-894, 2001.

[43] N. Oddershede and J. A. Jensen, "Effects influencing focusing in synthetic aperture vector flow imaging," IEEE Trans. Ultrason., Ferroelec., Freq. Contr., vol. 54, no. 9, pp. 1811-1825, 2007.

[44] J. A. Jensen, "Inter-leaved synthetic aperture sequences for measuring high vector flow velocities," in Proc. IEEE Ultrason. Symp., Oct. 2018.

[45] — "Estimation of high velocities in synthetic aperture imaging: I: Theory," IEEE Trans. Ultrason., Ferroelec., Freq. Contr., p. Submitted, 2018.

[46] $\longrightarrow$, "Estimation of high velocities in synthetic aperture imaging: II: Experimental investigation," IEEE Trans. Ultrason., Ferroelec., Freq. Contr., p. Submitted, 2018.

[47] M. C. Hemmsen, T. Kjeldsen, L. Lassen, C. Kjær, B. Tomov, J. Mosegaard, and J. A. Jensen, "Implementation of synthetic aperture imaging on a hand-held device," in Proc. IEEE Ultrason. Symp., 2014, pp. $2177-2180$

[48] J. A. Jensen and S. I. Nikolov, "Directional synthetic aperture flow imaging," IEEE Trans. Ultrason., Ferroelec., Freq. Contr., vol. 51, pp. 1107-1118, 2004.

[49] T. Di Ianni, C. V. Hoyos, C. Ewertsen, M. Nielsen, and J. A. Jensen, "High-frame-rate imaging of a carotid bifurcation using a lowcomplexity velocity estimation approach," in Proc. IEEE Ultrason. Symp., 2017, pp. 1-4.

[50] K. L. Hansen, J. Udesen, F. Gran, J. A. Jensen, and M. B. Nielsen, "Invivo examples of flow patterns with the fast vector velocity ultrasound method," Ultraschall in Med., vol. 30, pp. 471-476, 2009. 
[51] C. A. Villagomez-Hoyos, M. B. Stuart, K. L. Hansen, M. B. Nielsen, and J. A. Jensen, "Accurate angle estimator for high frame rate 2-D vector flow imaging," IEEE Trans. Ultrason., Ferroelec., Freq. Contr., vol. 63 , no. 6 , pp. 842-853, 2016.

[52] T. Di Ianni, T. Kjeldsen, C. Villagomez-Hoyos, J. Mosegaard, and J. A. Jensen, "Real-time implementation of synthetic aperture vector flow imaging in a consumer-level tablet," in Proc. IEEE Ultrason. Symp., 2017, pp. 1-4.

[53] J. P. Mynard and D. A. Steinman, "Effect of velocity profile skewing on blood velocity and volume flow waveforms derived from maximum Doppler spectral velocity," Ultrasound Med. Biol., vol. 39, no. 5, pp. 870-881, 2013.

\section{BIBLIOGRAPHIES}

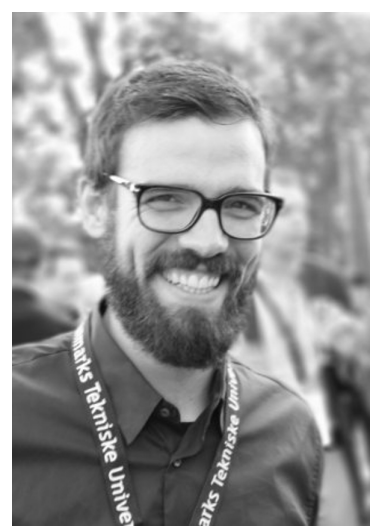

Tommaso Di Ianni received the B.Sc. and M.Sc. degrees in electronic engineering from the University of Bologna, Bologna, Italy, in 2011 and 2014, and the Ph.D. degree in biomedical engineering from the Technical University of Denmark, Kgs. Lyngby, Denmark, in 2017. He is currently a postdoctoral research fellow with the Department of Radiology, Stanford University School of Medicine, Stanford, CA, USA. His current research interests include ultrasoundmediated targeted drug delivery, contrast-enhanced ultrasound imaging, estimation of blood flow velocities, and synthetic aperture imaging techniques.

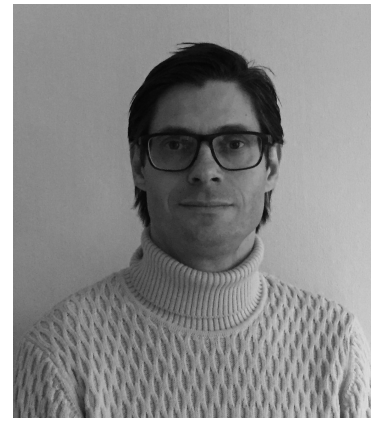

Kristoffer Lindskov Hansen received the M.D. and Ph.D. degree from Copenhagen University, Denmark, in 2003 and 2010, respectively, and became Medical Specialist in diagnostic radiology in 2014. He is currently with the Dept of Diagnostic Radiology, Copenhagen University Hospital, Denmark, and associate professor at Dept of Clinical Medicine, Copenhagen University, Denmark. His research concerns advanced ultrasound techniques with main focus on cardiac vector velocity estimation.

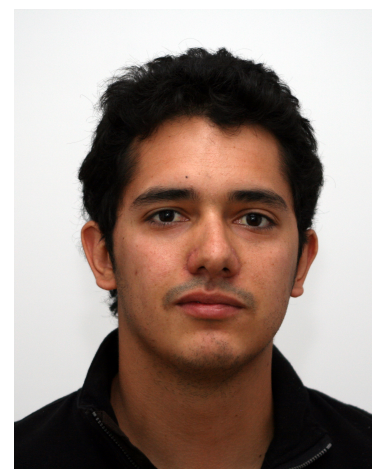

Carlos A. Villagmez Hoyos was in born 1985. He received his B.Sc. in electronic engineering during 2008, and M.Sc. degree in digital signal processing in January 2013 both from the National Autonomous University of Mexico. He spent six months at the Ultrasound Laboratory at the Federal University of Rio de Janiero in 2012. In 2016, he obtained his PhD degree in biomedical engineering at the Center for Fast Ultrasound Imaging at the Technical University of Denmark. The topic of his $\mathrm{PhD}$ research was "Synthetic aperture flow imaging". He is now with BK Medical.

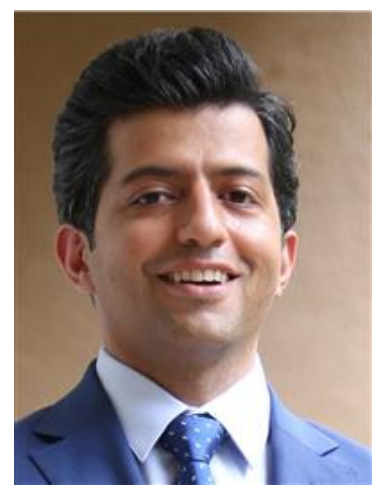

Ramin Moshavegh received the B.Sc. degree in electrical engineering from the Iran University of Science and Technology, Tehran, Iran, in 2006, the M.Sc. degree in biomedical engineering from the Chalmers University of Technology, Gothenburg, Sweden, in 2012, and the Ph.D. degree in biomedical engineering from the Center for Fast Ultrasound Imaging, Technical University of Denmark, Kongens Lyngby, Denmark, in 2016. From 2011 to 2014, he was an Image Analysis Researcher with the Medtech West, Gothenburg, Sweden. From 2017 to 2018, he was a Post-Doctoral Researcher with BK Ultrasound, Herlev, Denmark. He is currently a Senior Engineer in Research and Development, BK Ultrasound, where he facilitates the translation of research algorithms to commercially available products. 


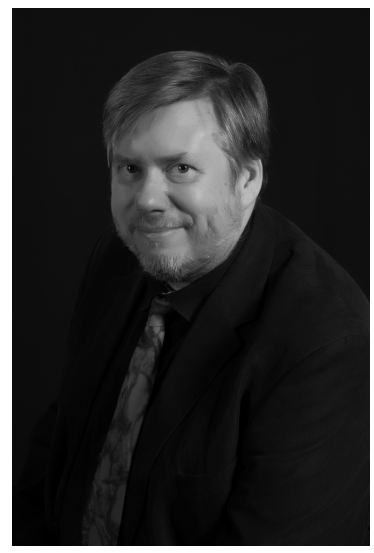

Michael B. Nielsen is a Medical graduate from the Faculty of Health Science, University of Copenhagen, Copenhagen, Denmark, in 1985, and received the Ph.D. degree in 1994, and the Dr.Med. Dissertation degree in 1998. He is a Full Professor of Oncoradiology with the University of Copenhagen, and a Consultant with the Department of Radiology, Rigshospitalet, Copenhagen. He has authored over 220 peer-reviewed journal articles on ultrasound or radiology. His current research interests include clinical testing of new ultrasound techniques, tumor vascularity, ultrasound elastography and training in ultrasound.

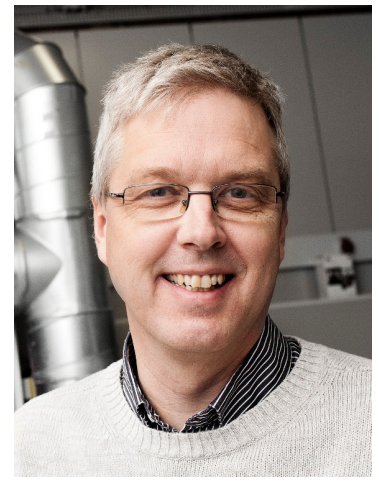

Jørgen Arendt Jensen earned his Master of Science in electrical engineering in 1985 and the $\mathrm{PhD}$ degree in 1989, both from the Technical University of Denmark. He received the Dr.Techn. degree from the university in 1996. He has since 1993 been full professor of Biomedical Signal Processing at the Technical University of Denmark at the Department of Electrical Engineering and head of Center for Fast Ultrasound Imaging since its inauguration in 1998. He has published more than 460 journal and conference papers on signal processing and medical ultrasound and the book "Estimation of Blood Velocities Using Ultrasound", Cambridge University Press in 1996. He is also the developer and maintainer of the Field II simulation program. He has been a visiting scientist at Duke University, Stanford University, and the University of Illinois at Urbana-Champaign. He was head of the Biomedical Engineering group from 2007 to 2010. In 2003 he was one of the founders of the biomedical engineering program in Medicine and Technology, which is a joint degree program between the Technical University of Denmark and the Faculty of Health and Medical Sciences at the University of Copenhagen. The degree is one of the most sought after engineering degrees in Denmark. He was chairman of the study board from 2003-2010 and adjunct professor at the University of Copenhagen from 2005-2010. He has given a number of short courses on simulation, synthetic aperture imaging, and flow estimation at international scientific conferences and teaches biomedical signal processing and medical imaging at the Technical University of Denmark. He has given more than 60 invited talks at international meetings, received several awards for his research, lately the Grand Solutions prize from the Danish Minister of Science and the order of Dannebrog by her Majesty the Queen. He is an IEEE Fellow since 2012. His research is centered around simulation of ultrasound imaging, 3-D synthetic aperture imaging, vector flow estimation, super resolution imaging, and construction of ultrasound research systems. 